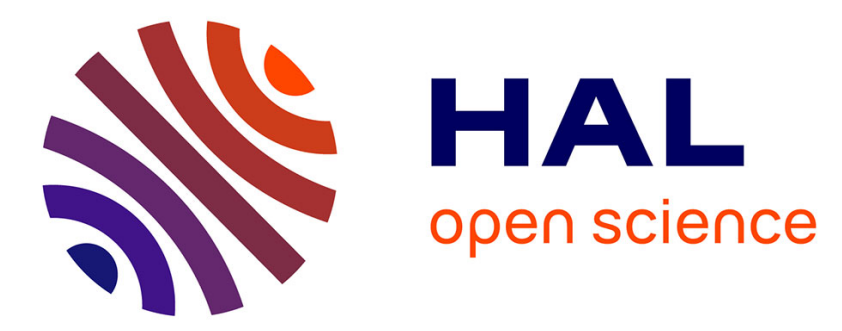

\title{
Les prébiotiques : une stratégie nutritionnelle pour prévenir des allergies
}

\author{
Amandine Sellé, Carole Brosseau, Sébastien Barbarot, Marie Bodinier
}

\section{To cite this version:}

Amandine Sellé, Carole Brosseau, Sébastien Barbarot, Marie Bodinier. Les prébiotiques : une stratégie nutritionnelle pour prévenir des allergies. Revue francaise d'allergologie, 2019, 59 (2), pp.112. 10.1016/j.reval.2018.10.007 . hal-02622824

\section{HAL Id: hal-02622824 \\ https://hal.inrae.fr/hal-02622824}

Submitted on 22 Oct 2021

HAL is a multi-disciplinary open access archive for the deposit and dissemination of scientific research documents, whether they are published or not. The documents may come from teaching and research institutions in France or abroad, or from public or private research centers.
L'archive ouverte pluridisciplinaire $\mathbf{H A L}$, est destinée au dépôt et à la diffusion de documents scientifiques de niveau recherche, publiés ou non, émanant des établissements d'enseignement et de recherche français ou étrangers, des laboratoires publics ou privés.

\section{다)(1) $(5$}

Distributed under a Creative Commons Attribution - NonCommerciall 4.0 International 


\title{
Les prébiotiques : une stratégie nutritionnelle pour prévenir des allergies.
}

\section{Prebiotics: a nutritional strategy to prevent allergies.}

\author{
A. Selle ${ }^{1}$, C. Brosseau ${ }^{1}$, S. Barbarot ${ }^{2,3} \&$ M. Bodinier ${ }^{1}$ \\ ${ }^{1}$ UR1268 BIA, INRA, Nantes, France \\ ${ }^{2}$ Service de Dermatologie, $\mathrm{CHU}$ de Nantes, Nantes, France \\ ${ }^{3}$ UMR PhAN, INRA, Nantes, France
}

\section{Résumé :}

Depuis les années 1970, les allergies connaissent une prévalence exponentielle. Elles représentent désormais un réel problème de santé publique, il est donc crucial de trouver une stratégie préventive non invasive efficace. Les allergies sont des maladies multifactorielles liées au dysfonctionnement de 3 acteurs biologiques: le microbiote, les muqueuses et le système immunitaire. Ces altérations conduisent à un défaut dans l'établissement de la tolérance immunitaire. Pour prévenir efficacement des allergies, il semble donc crucial d'agir sur l'homéostasie de ces 3 systèmes biologiques. L'alimentation joue un rôle essentiel pour notre santé, et notamment dans le développement de l'allergie. Les conditions environnementales durant la grossesse et les premiers jours de vie vont conditionner le développement de nos systèmes immuns, métaboliques, physiologiques et bactériens qui influenceront ensuite notre susceptibilité à développer des pathologies futures. II est de fait essentiel d'étudier l'impact des modifications de notre mode de vie à un stade précoce tel que notre régime alimentaire sur les fonctions immunes et le développement des maladies allergiques. Les prébiotiques, définis comme des ingrédients alimentaires, sont une piste d'investigation très intéressante dans ce contexte. Ils sont en effet capables d'agir sur le système immunitaire, le microbiote et la barrière intestinale. Deux stratégies de supplémentation avec les prébiotiques ont été décrites dans la littérature : en postnatal et en périnatal. Au sein de cette revue, nous nous attacherons à décrire les différents facteurs impliqués dans le développement des allergies et à démontrer l'importance de la périnatalité en tant que fenêtre d'intervention pour la prévention. Les prébiotiques seront ensuite définis 
ainsi que leurs mécanismes d'action. Enfin, l'ensemble des études précliniques et cliniques seront résumées pour évaluer l'efficacité d'une supplémentation en prébiotiques sur la prévention de l'allergie.

\section{Summary :}

Since 1970, allergies show an exponential growth. Today, they are a real public health problem. It is thus crucial to found a preventive non-invasive efficient strategy. Allergies are multifactorial diseases related to the dysfunction of 3 biological actors: the microbiota, the mucous membranes and the immune system. These alterations lead to a defect in the establishment of immune tolerance. To efficiently prevent allergies, it seems crucial to act on the homeostasis of these 3 biological systems. The diet plays an essential role in our health, especially in the development of allergy. The environmental conditions during pregnancy and the first days of life will condition the development of our immune, metabolic, physiological and bacterial systems which will then influence our susceptibility to develop future pathologies. It is truly essential to study the impact of lifestyle modifications at early stage such as our diet on immune functions and the development of allergic diseases. Prebiotics, defined as food ingredients, are a very interesting track of investigation in the context of allergies. They are able to act on the immune system, the microbiota and the intestinal barrier. Two strategies of prebiotic supplementation have been described in the literature: postnatal and perinatal. In this review, we will describe the various factors involved in the development of allergies and we will demonstrate the importance of perinatality as a window of intervention for prevention. Prebiotics will be then defined as well as their mechanisms of action. Finally, all preclinical and clinical studies will be summarized to evaluate the efficiency of prebiotic supplementation on allergy prevention. 


\section{Les facteurs responsables des allergies}

Les mécanismes de rupture de la tolérance conduisant à l'apparition de l'allergie ont largement été décrits dans la littérature (1), contrairement aux facteurs et mécanismes précoces responsables de la mise en place de la pathologie. Aujourd'hui, on estime que 30 à $40 \%$ de la population mondiale souffre d'une allergie (2). Cette augmentation drastique est notamment observée dans les pays industrialisés et s'accroît. Celle-ci semble corrélée au mode de vie occidental : l'exposition aux molécules polluantes en milieu urbain, les milieux de vie aseptisés, la consommation excessive d'antibiotiques et une alimentation pauvre en fibres et riche en graisses. L'hypothèse hygiéniste est une théorie selon laquelle l'ensemble de ces facteurs modifient le développement des paramètres immunitaires et microbiens conduisant à l'augmentation de la prévalence de l'allergies depuis les années 70 (3).

De plus, les nouvelles habitudes alimentaires dites « western diet » favorisent la dysbiose du microbiote chez l'adulte. Cette dysbiose est souvent observée chez les enfants allergiques, car le microbiote maternelle est directement transmis à l'enfant in utero et pendant l'accouchement (4) perturbant la maturation des systèmes immuns et muqueux.

- Impact du microbiote dans l'allergie (figure 1).

Trois hypothèses sont proposées pour expliquer la relation microbiote/allergies: la représentativité des souches bactériennes, les métabolites apportés par le microbiote et la stimulation inappropriée des pathogen recognition receptor (PRR). Bifidobacterium et Bacteroides sont deux bactéries à fort taux de fermentation produisant des acide gras à chaine courtes (AGCC) qui ont de nombreux effets bénéfiques (anti-inflammatoire, renforcement des barrières) (5). Une dysbiose en défaveur de ces bactéries fermenticides favoriserait le développement des allergies. Notamment, II a été démontré que les nouveau-nés atopiques âgés de 3 semaines présentaient un ratio Clostridium difficile/Bifidobacterium significativement plus élevé (6). Dans le cas de l'allergie respiratoire (AR), la dysbiose est également observable (7). La deuxième hypothèse concernant les effets des métabolites apportés par le microbiote a été très récemment confirmée chez l'homme. Le niveau fécal d'AGCC comme le pyruvate, l'acétate et le propionate est diminué chez des enfants atteints de dermatite atopique (DA) allergique. Le niveau d'AGCC produit par le microbiote jouerai donc un rôle dans le développement de DA suite à la sensibilisation allergique (8). La troisième hypothèse concerne l'immunité innée. Dès la naissance 
les enfants allergiques présentent une réponse accrue aux stimulations des récepteurs de l'immunité innée comme le TLR4 (toll like receptor 4) et le TLR9. Cette hyper-activation ne permet pas la maturation des lymphocytes $T$ CD4 $T_{H} 0$ en $T_{H} 1$ favorisant le développement de la réponse $T_{H} 2$, caractéristique de l'allergie. La dérégulation de la réponse inflammatoire dès la naissance pourrait donc avoir un rôle dans la polarisation anormale des lymphocytes $\mathrm{T}(9)$.

- Rôle des barrières physiologiques dans l'allergie (figure 1):

Les muqueuses intestinales, pulmonaires et cutanées ont un rôle de protection et d'absorption pour l'organisme. L'épithélium est composé de cellules épithéliales liées par des jonctions serrées formant une véritable barrière renforcée par une forte capacité de sécrétion augmentant l'étanchéité de ces barrières (mucus, sébum). Dans le cas des allergies, un dysfonctionnement des barrières est observé. II reste à ce jour difficile de démontrer si ces désordres physiologiques sont la cause ou la conséquence de l'allergie (10). Dans le cas des allergies cutanées ( $A C)$, on assiste à des modifications de perméabilité et de structure de la barrière cutanée. Ces défauts sont observables très tôt, notamment une forte perméabilité cutanée à 2 jours de vie est prédictive de l'apparition d'une DA à l'âge d'un an (11). Dans 50\% des cas, cette perméabilité est due à une mutation du gène de la filaggrine $(12,13)$. Dans le contexte des allergies alimentaires (AA), des modifications de perméabilité intestinale paracellulaire et transcellulaire sont aussi observées. L'augmentation de la perméabilité transcellulaire se caractérise par une surexpression du récepteur CD23 (récepteur de faible affinité pour les lgE) qui permet le transfert des complexes IgE/allergènes intacts. Ce passage des allergènes natifs vers les compartiments internes favorise ensuite la sensibilisation (14). Dans le cas des AR, l'inflammation pulmonaire est associée à l'atteinte de l'intégrité de l'épithélium pulmonaire correspondant à des lésions des jonctions serrées. Cette fragilité favorise ensuite l'exposition aux allergènes et l'inflammation chronique (15). Cette altération des épithelia est un facteur clé dans l'absorption de l'allergène et dans sa présentation aux cellules immunitaires.

- Altération du système immunitaire dans l'allergie (figure 1):

L'immunité à médiation cellulaire de type 2, riche en éosinophiles, basophiles, mastocytes, cellules $T$ CD4 de type $T_{H} 2$ et cellules lymphoïdes innées de type 2 (ILC2) protège l'hôte contre l'infection par les helminthes mais conditionne aussi le développement des allergies (16). Lors de l'exposition aux allergènes, les cellules 
épithéliales sont activées et produisent des chimiokines et des cytokines innées comme I'IL-1, I'IL-25, I'IL-33 et la lymphopoïétine stromale thymique (TSLP). Ces signaux vont permettent aux cellules dendritiques (DC) immatures d'augmenter l'expression des ligands OX40 et Notch, de diminuer la production d'IL-12 et de produire des chimiokines attirant les cellules $T_{H} 2$ telles que CCL17 et CCL22. Ces facteurs dérivés des cellules épithéliales vont aussi stimuler la prolifération et la survie des ILC2 et leur production d'IL-5 et IL-13. De même, les ILC2 sont recrutées très tôt au cours de la réponse à médiation cellulaire de type 2 et pourraient ainsi fournir de I'IL-13 pour stimuler la fonction des DC (principalement la migration) et favoriser les réponses de type $T_{H} 2$. L'IL-4 produite par les $T C D 4 T_{H} 2$ va induire la prolifération des lymphocytes $\mathrm{B}$ ainsi que la commutation de classe isotopique $\lg \mathrm{E}$, signature de l'allergie (17). Les mécanismes listés ci-dessus sont non exhaustifs. L'excellente revue de Rothenberg et al. complète le sujet en décrivant plus précisément les mécanismes impliqués (18).

\section{Période périnatale: étape critique pour la mise en place des systèmes biologiques}

L'environnement et les facteurs héréditaires jouent un rôle majeur sur le développement des systèmes microbiens, immuns et des muqueuses (19). Ces systèmes se mettent en place in utéro et les 1000 premiers jours de vie (20). Des échanges de facteurs immuns et microbiens, entre la mère et le fœtus et /ou le nouveau-né se font in utero par le sang de cordon, lors de l'accouchement par la flore vaginale et pendant l'allaitement par le lait. La période périnatale semble donc une étape clé pour moduler les systèmes biologiques et ainsi impacter sur le développement de l'allergie.

Le microbiote primaire s'installe dès la naissance de l'enfant, par un transfert de flore vaginale de la mère à l'enfant. Cette colonisation lui confère les espèces pionnières comme Escherichia coli/Shigella, Bacteroides, Bifidobacterium et Lactobacillus. Ce premier microbiote joue un rôle important pour la santé de l'hôte car il est impliqué dans la mise en place de nombreuses fonctions immunitaires, métaboliques et physiologiques qui sont cruciales pour le bon fonctionnement de l'organisme(21). Par conséquent, une stratégie nutritionnelle visant à instaurer un microbiote diversifié et 
équilibré pour favoriser le développement de ces systèmes biologiques représente une stratégie de choix pour prévenir l'allergie.

Ainsi, plusieurs études ont mis en place des stratégies nutritionnelles en périnatale et/ou en postnatale capables de moduler favorablement le microbiote et le système immunitaire (22). Dans le cas de l'allergies, des antioxydants (vitamine $\mathrm{C}$, vitamine $\mathrm{E}$, B-carotène, zinc), des folates, de la vitamine $D$, des omégas 3 , des probiotiques et des prébiotiques ont été testés (23). Les prébiotiques représentent donc des outils capables de moduler la composition microbienne et la production de métabolites importants pour la croissance de certaines bactéries résidentes dans notre intestin.

\section{Les prébiotiques}

- Définitions

Les prébiotiques sont décrits pour la première fois par Gibson et Roberfroid en 1995 comme « des ingrédients alimentaires non digestibles influençant de manière bénéfique la santé de l'hôte en stimulant l'activité d'une ou plusieurs bactéries commensales du côlon »(24). L'acquisition de nouvelles données scientifiques sur leur mode d'action et leur spécificité a permis d'affiner cette définition, ils sont alors requalifiés en 2017 par l'Association scientifique international des probiotiques et prébiotiques (ISAPP) comme « des substrats sélectivement utilisés par les microorganismes de l'hôte conférant des bénéfices pour sa santé ». Les prébiotiques doivent remplir 3 critères : 1 : être résistants à la digestion dans l'estomac et la partie haute de l'intestin, 2 : fermentescibles par le microbiote de l'intestin et 3 : stimuler spécifiquement la croissance et/ou l'activité des bactéries intestinales bénéfiques pour notre santé (25). Cependant, les bénéfices des prébiotiques ne sont pas réduits exclusivement à l'intestin, ils peuvent également agir de manière systémique dans certains traitements thérapeutiques $(26,27)$.

Les prébiotiques sont des substrats alimentaires non viables contrairement aux probiotiques. Ils sont généralement composés de sucres liés comme les oligosaccharides et les polysaccharides à chaînes courtes. Ces molécules ont pour caractéristique chimique d'être indigestibles par les enzymes présentes dans le tractus intestinal. Elles vont donc pouvoir servir de substrats nutritionnels aux microorganismes considérés «bénéfiques» comme Bifidobacterium et Bacteroïdes ou entrer en contact directement avec les cellules environnantes (28). 
Les fructanes tels que les fructo-oligosaccharides (FOS) et l'inuline, ainsi que les galactanes comme les galacto-oligosaccharides (GOS) ont été les prébiotiques les plus étudiés pour leurs effets modulateurs sur le microbiote. Aujourd'hui de nouveaux composés sont considérés comme des prébiotiques : les oligosaccharides présents dans le lait humain (Human milk oligosaccharides: HMO), de nouveaux oligosaccharides comme les acides gras polyinsaturés ou encore les acides linoléiques conjugués (25). Les prébiotiques les plus présents dans nos aliments sont les FOS et l'inuline, retrouvés dans certains légumes comme le poireau, l'oignon, l'ail, l'artichaut, la chicorée ou l'asperge, et dans certains fruits comme la banane et dans des céréales.

Les FOS, GOS et l'inuline sont présents dans certaines préparations pour nourrissons. En Europe, la consommation de prébiotiques naturels dans le cadre d'une alimentation équilibrée est estimée entre 3 et 11 gramme/jour et par personne (29). Les effets des prébiotiques sont souvent confondus avec les effets d'une consommation de fibres dû à une confusion quant à leurs définitions. En effet, la plupart des fibres alimentaires sont faiblement digestibles et influencent de manière globale l'ensemble du microbiote de l'intestin, contrairement aux prébiotiques qui eux ont un effet s'appliquant exclusivement à certaines espèces, qu'on appelle bifidogènes (28). Enfin, les prébiotiques vont avoir un effet sur le fonctionnement de différents systèmes comme le microbiote, les muqueuses et le système immunitaire par différents mécanismes.

- Les mécanismes d'action des prébiotiques

Les prébiotiques peuvent influencer l'organisme de deux façons (figure 2). Ils peuvent être utilisés de manière indirecte comme substrat pour certaines bactéries commensales et les produits de cette fermentation, les AGCC, peuvent ensuite influencer les différents processus moléculaires et cellulaires des tissus de l'hôte. De nouvelles recherches suggèrent que les prébiotiques peuvent agir de manière directe en se fixant sur les récepteurs des cellules cibles.

Les effets indirects médiés par les acides gras à chaînes courtes :

La majorité des effets indirects des prébiotiques sont médiés par les AGCC comme le butyrate, le pyruvate, l'acétate et le propionate. En effet, à l'issue de la fermentation microbienne des prébiotiques, des AGCC sont produits dans la lumière intestinale. Ils entrent alors en contact avec les cellules environnantes telles que les 
cellules épithéliales et les cellules de l'immunité innée et adaptative pour stimuler certaines voies de signalisation. Ces métabolites vont se fixer aux récepteurs couplés aux protéines $G$ ( $G$ protein-coupled receptor GPR) comme FFAR2, FFAR3, GPR109a et induire une voie de signalisation qui à l'heure actuelle n'est pas clairement définie (30). Cela a pour conséquence de moduler l'action d'enzymes et de facteurs de transcription comme les histones déacétylases et l'hypoxia-inducible facteur (30) qui vont jouer un rôle sur la régulation de l'expression des gènes. Singh et son équipe ont montré que les AGCC orientaient les lymphocytes $T$ naïfs vers un profil tolérogène par la stimulation des $D C$. Ce mécanisme est médié par la liaison du butyrate au GPR109A présent sur les DC, permettant l'expression des protéines 110 et Aldh1a1 essentielles à la différenciation des lymphocyte $T$ régulateurs (31). Trompette et al., ont également montré que le propionate : $1 /$ module favorablement le microbiote pulmonaire et intestinal en augmentant la proportion de Firmicutes et Bacteroides ; 2/ diminue l'hématopoïèse des DC stimulatrices de la réponse $T_{H} 2$. Ces mécanismes médiés par le propionate conduisent à une réduction de I'inflammation au cours de la réaction allergique et à une diminution des symptômes de l'asthme (32). Les prébiotiques remodèlent l'homéostasie microbienne de l'intestin en augmentant la sécrétion d'AGCC par le microbiote. En particulier l'acétate et le butyrate améliorent la tolérance orale et protègent de l'allergie alimentaire en augmentant l'activité rétinal déshydrogénase des DC $\mathrm{CD}_{103^{+}}$. Cette protection dépend de la vitamine $A$ présente dans l'alimentation. Ce régime alimentaire stimule également la production d'IgA impliquées dans la tolérance. Les souris dépourvues du GPR43 ou du GPR109A, récepteurs pour les AGCC, présentent une allergie alimentaire exacerbée et moins de $\mathrm{DC} C D 103^{+}$. Ces apports diététiques, y compris les fibres et la vitamine $A$, régulent donc de nombreuses voies protectrices dans le tractus gastro-intestinal, nécessaires à la tolérance aux antigènes alimentaires (33).

\section{Les effets direct des prébiotiques sur l'organisme :}

\section{$\checkmark$ La modulation du microbiote:}

Les prébiotiques sont principalement connus pour leur impact sur la modulation du microbiote intestinal. Plus que la composition, c'est surtout le nombre de bactéries qui est modifié. II est admis que les bactéries des taxons Lactobacille et Bifidobacterium sont significativement plus représentés à des doses moyennes de 58 grammes/jour de prébiotiques. L'expansion des bactéries bénéfiques empêche 
l'implantation des bactéries pathogènes et réduit le nombre de certaines bactéries commensales par effet de compétition $(34,35)$. Hopkins et son équipe ont montré que la croissance des Bifidobactéries suite à la consommation de prébiotiques est corrélée avec une diminution de la population de Clostridium difficile (36). Dans un deuxième temps, les bactéries vont également augmenter leur production de peptides antimicrobiens empêchant la colonisation de l'épithélium intestinale par les pathogènes (37).

$\checkmark \quad$ Interaction avec les cellules épithéliales

Lors de l'ingestion de prébiotiques, les oligosaccharides pénètrent dans l'intestin. Ils entrent alors en contact direct avec les cellules épithéliales. Lors de l'étude in vitro de l'effet anti-inflammatoire des prébiotiques sur des lignées d'entérocytes, Marwa Zenhom et al. ont mis en évidence la fixation des oligosaccharides aux récepteurs «peptidoglycane recognition protein (PGIYRP) ». Cette liaison inhibe l'expression de cytokines pro-inflammatoires et la translocation du facteur NF-kB (nuclear factor kappa-light-chain-enhancer of activated B cells) dans le noyau. L'effet antiinflammatoire est médié par la signalisation de PPARy qui induit l'expression de PGIYRP3 produisant un effet anti-inflammatoire. De plus l'ajout de prébiotiques au milieu de culture augmente la production de cytokine (IL-8) et de chimiokines (CCL1 et CXCL2, CXCL1) par la liaison du TLR4 (38). En effet, les cellules épithéliales des souris déficientes en TLR4 ont une réponse nettement diminué aux prébiotiques. Les prébiotiques sont donc des agonistes du TLR4 (39). Une troisième étude a permis de mettre en évidence les interactions entre les cellules épithéliales et les DC via le prébiotique. L'ajout de prébiotique dans le milieu de culture de DC ne modifie pas leur sécrétion. Par contre, l'ajout de surnageant de culture de cellules épithéliales incubées avec des prébiotiques sur des DC augmente le ratio de sécrétion IL-10/IL12 par les DC. Cela suggère que les prébiotiques induisent des DC tolérogènes et que l'effet immunomodulateur induit par les prébiotiques est médié par les molécules sécrétées par les cellules épithéliales. Les molécules secrétées par les cellules épithéliales et les DC au contact des prébiotiques jouent aussi un rôle sur la stimulation des lymphocytes T CD4+. Les GOS sont capables d'augmenter la sécrétion de l'IL-10 par les T régulateurs. L'inuline quant à elle, diminue la réponse T CD4+ de type $\mathrm{T}_{\mathrm{H}} 2$ (40). Dans le cadre d'une étude sur les symbiotiques, S. De Kivit et ses collègues ont montré que l'ingestion de prébiotiques GOS/FOS seuls ou combinés avec Bifidobacterium breve $M-16 \mathrm{~V}$, induit une sécrétion de galectine-9 par 
les cellules épithéliales de l'intestin. La sécrétion de galectine-9 diminue la dégranulation des mastocytes et favorise les réponses $T_{H} 1$ et $T$ régulatrice. Cependant à ce jour, les mécanismes d'interaction des prébiotiques avec les DC ou les lymphocytes $T$ ne sont pas bien élucidés $(41,42)$. L'ensemble de ces études montrent que les prébiotiques peuvent entrer directement en contact avec les cellules de l'épithélium intestinal sans passer par le microbiote. Par la voie de signalisation du TLR4, et des PGIYRP ils induisent une sécrétion de cytokines antiinflammatoires influençant le profil des cellules avoisinantes comme les cellules immunitaires sous-jacentes. En plus du contact avec les cellules épithéliales, les GOS et FOS sont capables de franchir la monocouche de cellules épithéliales in vitro ce qui indique qu'ils pourraient entrer en contact avec les cellules immunitaires se trouvant dans l'épithélium intestinal et induire potentiellement d'autres effets (43).

\section{$\checkmark$ Interaction avec les cellules du système immunitaire}

Certains prébiotiques semblent être absorbés au niveau de la barrière intestinale et peuvent se retrouver ainsi en contact avec les cellules du système immunitaire circulantes. L'inuline et les FOS induisent la sécrétion d'IL-10, de CCL1 et du TNFa (tumor necrosis factor $\alpha$ ) par les monocytes sanguins. Cette sécrétion est due à l'activation de la voie NF-kB par la liaison du récepteur TLR4 activant la voie des MAPK (mitogen activated protein kinase) et PI3K (phosphatidylinositol-3-kinases). Au contraire les FOS et l'inuline n'ont pas d'impact significatif sur la sécrétion de cytokines par les lymphocytes $T$ (47). Sur les DC issus de monocytes sanguins humains, les GOS et FOS induisent une sécrétion d'IL-10 stimulée par la liaison du TLR4. Au contraire, IL-12 n'est pas détectable. L'augmentation de la sécrétion d'IL10 par les $D C$ va avoir pour conséquence d'induire des lymphocytes $T$ régulateurs (44). Ces études montrent que les prébiotiques ont un effet anti-inflammatoire sur les DC via la liaison des prébiotiques au TLR4. L'interaction des prébiotiques avec les cellules immunitaires n'est pas totalement comprise, mais il est évident que les prébiotiques peuvent orienter les réponses $T_{H} 1, T_{H} 2$ et $T$ régulatrice. Les effets tolérogènes et la modulation de flore commensale suite à l'ingestion de prébiotiques est encourageante pour prévenir le développement des allergies. Notamment, la mise en place d'une stratégie nutritionnelle contenant des prébiotiques semble une possibilité intéressante à envisager. Plusieurs modèles précliniques et cliniques sont déjà publiés. 


\section{L'utilisation des prébiotiques pour prévenir les allergies}

- Etudes précliniques

A partir du modèle animal, plusieurs équipes de recherche ont mis en place des stratégies nutritionnelles utilisant différents prébiotiques pour évaluer leur impact sur le développement des allergies (tableau 1). Les prébiotiques ont été donnés soit à la mère soit à la descendance à différents moment de vie ou de gestation. La supplémentation a été suivie par l'induction d'un modèle d'allergie et les paramètres immunitaires, microbiens et physiologiques ont été évalués.

\section{Etudes en postnatal}

Les travaux de J. Watanabe montrent qu'un régime enrichi en FOS pendant 3 semaines sur des souris âgées de 5 semaines modifie qualitativement le microbiote intestinal. Le nombre de Bifidobacterium intestinales est augmenté en corrélation avec une diminution de la sévérité de l'allergie. Cela suggère que la consommation de prébiotiques par les Bifidobacterium est responsable de la diminution des symptômes allergiques (45). D'autre part, l'observation des paramètres immuns montre que la supplémentation en GOS de souris âgées de 4 semaines pendant 14 jours réduit l'infiltration péritonéale de cellules impliquées dans l'inflammation due à une diminution de la sécrétion de chimiokines (46). La consommation de FOS pendant 8 semaines par des souris âgées de 6 semaines réduit de manière significative le nombre de mastocytes et le taux de formation d'œdème dans le duodénum lors de la mise en place de l' allergie alimentaire (47). Vos et al. montrent qu'un régime supplémenté par un mélange GOS/FOS pendant 8 semaines par des souris âgées de 5 à 8 semaines diminue l'asthme allergique en inhibant la réponse $\mathrm{T}_{\mathrm{H}} 2$ et en induisant l'activation de la réponse $\mathrm{T}_{\mathrm{H}}$ 1 (48). L'ensemble de ces données suggèrent que les prébiotiques ont un rôle important dans la prévention de l'allergie chez l'adulte et l'enfant. Une intervention pendant la mise en place des grands systèmes biologiques telle que la grossesse et ou l'allaitement, représente aussi une stratégie très prometteuse.

\section{Etudes en périnatale :}

Une supplémentation maternelle de GOS/FOS influence le développement du système immunitaire de la descendance en modulant les paramètres immunitaires maternels et fœtaux (49). La supplémentation lors de la lactation entraîne moins de 
symptômes allergiques chez la progéniture indépendamment du fait que la mère ait été sensibilisée à l'allergène avant l'accouplement (49). L'exposition aux prébiotiques GOS/inuline pendant la gestation et la lactation entraine une diminution de la réaction d'allergie alimentaire au blé. Cet effet protecteur est mesuré par une réduction des symptômes cliniques associé à un renforcement de la perméabilité intestinale et à une modification du microbiote (50). L'administration de prébiotiques (GOS/ inuline) à des souris pendant la période périnatale puis post-sevrage stimule les voies liées à la tolérance (réponses $T$ régulatrice, $T_{H} 1$ et inhibition de la production d'IL-17A) et renforce la barrière intestinale (expressions fortes de HSP-25, MUC-2 et ZO-1) (50). L'administration précoce des prébiotiques favorise également un maintien des voies de la tolérance sur un modèle de souris allergiques au blé (51). Cependant, on ne retrouve pas ces effets favorables lorsque que les prébiotiques sont donnés uniquement après le sevrage. Ces résultats suggèrent que la gestation, la mise bas et l'allaitement pourraient être des périodes clés durant lesquelles les réponses immunitaires peuvent être significativement modulées. Fujiwara montre également que la consommation de FOS par la mère et les petits diminue la sévérité de la DA chez la progéniture (52). Ainsi Bouchaud et al., confirment qu'une supplémentation en GOS/Inuline pendant la gestation et l'allaitement protège d'une altération de la barrière intestinale, induit un environnement tolérogène et modifie le micobiote chez les descendants (53). Ces résultats suggèrent que la modulation du microbiote intestinal par les prébiotiques tôt dans la vie peut être bénéfique dans la prévention de l'allergie. L'exposition périnatale aux prébiotiques par la mère dans des modèles animaux a des effets régulateurs sur le système immunitaire et sur la diversité du microbiote. Cela permet de prévenir des maladies allergiques de la descendance. II existerait donc une transmission de la mère au fœtus/nourrisson de certains facteurs biologiques protecteurs induits par la consommation de prébiotiques. Cet effet antiallergique semble se perpétuer dans le temps protégeant la descendance.

- Etudes cliniques

A l'issus de ces études précliniques, plusieurs essais cliniques ont été réalisées (tableau 2) avec les prébiotiques mais uniquement en intervenant chez l'enfant. Deux méta-analyses recensent l'ensemble des études utilisant une supplémentation alimentaire en prébiotiques (FOS/GOS, polidextrose, GOS/FOS dans la plupart des cas) dans les préparations infantiles chez les jeunes enfants pour prévenir les 
symptômes allergiques $(54,55)$. 6 études ont observé que la supplémentation en prébiotique diminue le risque de développer de l'eczéma, mais l'intervalle de confiance ne permet pas d'exclure l'effet nul (56-60). 2 études montrent une légère diminution des risques de développement de l'asthme et seulement une étude montre la réduction de l'AA $(56,58)$. Néanmoins, la caractérisation de l'AA n'est pas suffisamment précise pour confirmer l'effet préventif (58). En conclusion, les prébiotiques administrés directement à l'enfant semblent prévenir de l'allergie. Néanmoins, les problèmes de caractérisation de l'allergie, l'hétérogénéité des prébiotiques utilisés ainsi que la fiabilité des études ne permette pas de conclure sur un réel effet préventif. Aucune donnée n'est disponible à ce jour, en clinique, concernant les effets d'une supplémentation maternelle en prébiotiques pendant la grossesse et/ou l'allaitement pour prévenir de l'allergie chez l'enfant à risque. Cependant, deux études cliniques sont en cours dans ce contexte : SYMBA (numéro d'identification de l'essai: ACTRN12615001075572) et PREGRALL (numéro d'identification de l'essai: NCT03183440).

\section{Conclusion}

L'incidence des allergies augmente de façon dramatique dans les pays industrialisés et aucun moyen préventif chez l'adulte et l'enfant n'a pour le moment été établi. Les allergies sont dues à des altérations du fonctionnement et/ou de composition du système immunitaire, des muqueuses et du microbiote. Notamment les patients atteints d'allergies montrent: 1/ un dysfonctionnement accru des muqueuses cutanées, pulmonaires et intestinales ; 2/ une dysbiose des microbiotes associée en faveur d'espèces comme les Firmicutes et en défaveur des Bifidobactéries ; $3 /$ une hyperstimulation des réponses $T_{H} 2$ face à un antigène au départ inoffensif. Ces 3 grands systèmes se mettent en place in utero et les premiers jours de vie (fenêtre périnatale) puis ils évoluent une fois adulte. Au cours de la périnatalité, il existe des échanges fondamentaux entre la mère et son enfant. Ainsi, pendant la grossesse on assiste à des transferts immuns et microbiens de la mère au fœtus via le sang de cordon, lors de l'accouchement la flore vaginale de la mère est transférée au nourrisson et enfin lors de l'allaitement de nombreux facteurs immuns et microbiens sont ingérés par l'enfant via le lait maternel. Ces transferts vont faire maturer les systèmes biologiques de l'enfant qui vont ensuite conditionner sa susceptibilité à 
développer des pathologies comme les allergies. Les allergies (cutanées ou alimentaires) se détectent parfois très tôt dans la vie, dès le premier mois de naissance indiquant qu'il existe d'ores et déjà des dysfonctionnements de ces systèmes biologiques. La période périnatale est donc une fenêtre très intéressante pour prévenir le développement d'allergie chez les nouveau-nés. Les prébiotiques sont des ingrédients alimentaires issus de fibres favorisant certaines espèces bénéfiques du microbiote intestinal. Les bactéries fermentent ces molécules et produisent des AGCC capables de moduler la transcription de gènes vers un profil anti-inflammatoire. Les prébiotiques agissent également directement sur les cellules épithéliales de l'intestin et le système immunitaire associé, produisant un effet antiallergique. À l'instar de ces découvertes, des études précliniques encourageantes montrent que quelle que soit la période d'exposition, les prébiotiques ont un effet préventif des allergies. Les études cliniques effectuées en postnatal montrent des résultats trop hétérogènes mais encourageants. II est nécessaire d'obtenir des données cliniques sur la supplémentation maternelle en prébiotiques. Malgré le manque de données, l'Organisation mondiale de l'allergie recommande d'utiliser les prébiotiques chez les nourrissons qui ne sont pas allaités exclusivement, qu'ils soient à haut ou à faible risque de développer des allergies (61). 


\section{REFERENCES}

1. Sampson HA, O'Mahony L, Burks AW, Plaut M, Lack G, Akdis CA. Mechanisms of food allergy. J Allergy Clin Immunol. 2018;141(1):11-9.

2. Prescott SL. Early-life environmental determinants of allergic diseases and the wider pandemic of inflammatory noncommunicable diseases. J Allergy Clin Immunol. 2013;131(1):23-30.

3. Prescott S, Allen KJ. Food allergy: Riding the second wave of the allergy epidemic. Pediatr Allergy Immunol. 2011;22(2):155-60.

4. Neu J, Rushing J. Cesarean versus vaginal delivery: long term infant outcomes and the hygiene hypothesis. Clin Perinatol. 2012;38(2):321-31.

5. Song H, Yoo Y, Hwang J, Na YC, Kim HS. Faecalibacterium prausnitzii subspecies-level dysbiosis in the human gut microbiome underlying atopic dermatitis. J Allergy Clin Immunol. 2016;137(3):852-60.

6. Kalliomäki M, Kirjavainen P, Eerola E, Kero P, Salminen S, Isolauri E. Distinct patterns of neonatal gut microflora in infants in whom atopy was and was not developing. J Allergy Clin Immunol. 2001;107(1):129-34.

7. Arrieta M-C, Stiemsma LT, Dimitriu PA, Thorson L, Russell S, Yurist-Doutsch $S$, et al. Early infancy microbial and metabolic alterations affect risk of childhood asthma. Sci Transl Med. 2015;7(307):1-14.

8. Le Duc Huy Ta. Comparative Analysis of Fecal Short Chain Fatty Acids Profiles in Atopic Dermatitis And Healthy Infants. J Allergy Clin Immunol. 2018;141(2).

9. Tulic MK, Hodder M, Forsberg A, McCarthy S, Richman T, DVaz N, et al. Differences in innate immune function between allergic and nonallergic children: New insights into immune ontogeny. J Allergy Clin Immunol. 2011;127(2):470-8.

10. Mattila P, Joenväärä S, Renkonen J, Toppila-Salmi S, Renkonen R. Allergy as an epithelial barrier disease. Clin Transl Allergy. 2011;1(1):1-8.

11. Kelleher M, Dunn-Galvin A, Hourihane JOB, Murray D, Campbell LE, McLean WHI, et al. Skin barrier dysfunction measured by transepidermal water loss at 2 days and 2 months predates and predicts atopic dermatitis at 1 year. J Allergy Clin Immunol. 2015;135(4):930-5.

12. O'Regan GM, Irvine AD. The role of filaggrin in the atopic diathesis. Clin Exp Allergy. 2010;40(7):965-72. 
13. Ogrodowczyk A, Markiewicz L, Wróblewska B. Mutations in the filaggrin gene and food allergy. Prz Gastroenterol. 2014;9(4):200-7.

14. Heyman M. Gut barrier dysfunction in food allergy. Eur J Gastroenterol Hepatol. 2005;17(12):1279-85.

15. Wittekindt $\mathrm{OH}$. Tight junctions in pulmonary epithelia during lung inflammation. Pflugers Arch Eur J Physiol. 2017;469(1):135-47.

16. Hammad H, Lambrecht BN. Barrier Epithelial Cells and the Control of Type 2 Immunity. Immunity [Internet]. 2015;43(1):29-40. Available from: http://dx.doi.org/10.1016/j.immuni.2015.07.007

17. Hussain M, Borcard L, Walsh KP, Pena Rodriguez M, Mueller C, Kim BS, et al. Basophil-derived IL-4 promotes epicutaneous antigen sensitization concomitant with the development of food allergy. J Allergy Clin Immunol. 2018;141(1):22334.

18. Rothenberg ME, Saito $H$, Peebles RS. Advances in mechanisms of allergic disease in 2016. J Allergy Clin Immunol. 2017;140(6):1622-31.

19. Alma J Nauta, Kaouther Ben Amor, Jan Knol, Johan Garssen and E van der B. Relevance of pre- and postnatal nutrition to development and interplay between themicrobiota and metabolic and immune systems. Am J Clin Nutr. 2013;98(1):586S-593S.

20. Wopereis H, Oozeer R, Knipping K, Belzer C, Knol J. The first thousand days intestinal microbiology of early life: Establishing a symbiosis. Pediatr Allergy Immunol. 2014;25(5):428-38.

21. Dominguez-Bello MG, Costello EK, Contreras M, Magris M, Hidalgo G, Fierer $\mathrm{N}$, et al. Delivery mode shapes the acquisition and structure of the initial microbiota across multiple body habitats in newborns. Proc Natl Acad Sci. 2010;107(26):11971-5.

22. Heine RG. Food Allergy Prevention and Treatment by Targeted Nutrition. Ann Nutr Metab. 2018;72(suppl 3):33-45.

23. Szajewska $\mathrm{H}$. Early nutritional strategies for preventing allergic disease. Isr Med Assoc J. 2012;14(1):58-62.

24. Gibson, Roberfroid MB. Dietary Modulation of the Human Colonie Microbiota $\square$ : Introducing the Concept of Prebiotics. J Nutr. 1995;125(6):1401-12.

25. Gibson GR, Hutkins R, Sanders ME, Prescott SL, Reimer RA, Salminen SJ, et al. Expert consensus document: The International Scientific Association for 
Probiotics and Prebiotics (ISAPP) consensus statement on the definition and scope of prebiotics. Nat Rev Gastroenterol Hepatol. 2017;14(8):491-502.

26. Gregor Reid. Molecular Methods to Measure Intestinal Bacteria: A Review. J AOAC. 2012;95(1):5-24.

27. Kaczmarczyk, Melissa M., Miller, Michael J., Freund GG. The health benefits of dietary fiber: beyond the usual suspects of type 2 diabetes, cardiovascular disease and colon cancer. Metabolism. 2013;61(8):1058-66.

28. Bindels LB, Delzenne NM, Cani PD, Walter J. Opinion: Towards a more comprehensive concept for prebiotics. Nat Rev Gastroenterol Hepatol. 2015;12(5):303-10.

29. Braegger C. Prébiotiques. Paediatrica. 2004;15(6):22-3.

30. Corrêa-Oliveira R, Fachi JL, Vieira A, Sato FT, Vinolo MAR. Regulation of immune cell function by short-chain fatty acids. Clin Transl Immunol. 2016;5(4):73.

31. Singh N, Gurav A, Sivaprakasam S, Brady E, Padia R, Shi H, et al. Activation of Gpr109a, receptor for niacin and the commensal metabolite butyrate, suppresses colonic inflammation and carcinogenesis. Immunity. 2014;40(1):128-39.

32. Trompette A, Gollwitzer ES, Yadava K, Sichelstiel AK, Sprenger N, Ngom-Bru $\mathrm{C}$, et al. Gut microbiota metabolism of dietary fiber influences allergic airway disease and hematopoiesis. Nat Med. 2014;20(2):159-66.

33. Tan J, McKenzie C, Vuillermin PJ, Goverse G, Vinuesa CG, Mebius RE, et al. Dietary Fiber and Bacterial SCFA Enhance Oral Tolerance and Protect against Food Allergy through Diverse Cellular Pathways. Cell Rep. 2016;15(12):2809_ 24.

34. Slavin J. Fiber and prebiotics: Mechanisms and health benefits. Nutrients. 2013;5(4):1417-35.

35. Vandeputte D, Falony G, Vieira-Silva S, Wang J, Sailer M, Theis S, et al. Prebiotic inulin-type fructans induce specific changes in the human gut microbiota. Gut. 2017;66(11):1968-74.

36. Hopkins MJ, Macfarlane GT. Nondigestible oligosaccharides enhance bacterial colonization resistance against Clostridium difficile in vitro. Appl Environ Microbiol. 2003;69(4):1920-7.

37. Kawai T, Akira S. Toll-like Receptors and Their Crosstalk with Other Innate 
Receptors in Infection and Immunity. Immunity. 2011;34(5):637-50.

38. Zenhom M, Hyder A, de Vrese M, Heller KJ, Roeder T, Schrezenmeir J. Prebiotic Oligosaccharides Reduce Proinflammatory Cytokines in Intestinal Caco-2 Cells via Activation of PPAR and Peptidoglycan Recognition Protein 3. J Nutr. 2011;141(5):971-7.

39. Capitan-Canadas F, Ortega-Gonzalez M, Guadix E, Zarzuelo A, Suarez MD, de Medina FS, et al. Prebiotic oligosaccharides directly modulate proinflammatory cytokine production in monocytes via activation of TLR4. Mol Nutr Food Res. 2014;58(5):1098-110.

40. Bermudez-Brito M, Sahasrabudhe NM, Rösch C, Schols HA, Faas MM, De Vos $P$. The impact of dietary fibers on dendritic cell responses in vitro is dependent on the differential effects of the fibers on intestinal epithelial cells. Mol Nutr Food Res. 2015;59(4):698-710.

41. De Kivit S, Saeland E, Kraneveld AD, Van De Kant HJG, Schouten B, Van Esch BCAM, et al. Galectin- 9 induced by dietary synbiotics is involved in suppression of allergic symptoms in mice and humans. Allergy Eur J Allergy Clin Immunol. 2012;67(3):343-52.

42. de Kivit S, Kostadinova AI, Kerperien J, Morgan ME, Muruzabal VA, Hofman GA, et al. Dietary, nondigestible oligosaccharides and Bifidobacterium breve M$16 \mathrm{~V}$ suppress allergic inflammation in intestine via targeting dendritic cell maturation. J Leukoc Biol. 2017;102(1):105-15.

43. Eiwegger T, Stahl B, Haidl P, Schmitt J, Boehm G, Dehlink E, et al. Prebiotic oligosaccharides: In vitro evidence for gastrointestinal epithelial transfer and immunomodulatory properties. Pediatr Allergy Immunol. 2010;21(8):1179-88.

44. Lehmann S, Hiller J, Van Bergenhenegouwen J, Knippels LMJ, Garssen J, Traidl-Hoffmann C. In vitro evidence for immune-modulatory properties of nondigestible oligosaccharides: Direct effect on human monocyte derived dendritic cells. PLoS One. 2015;10(7):1-15.

45. Watanabe J, Sasajima N, Aramaki A, Sonoyama K. Consumption of fructooligosaccharide reduces 2,4-dinitrofluorobenzene-induced contact hypersensitivity in mice. Br J Nutr. 2008;100(2):339-46.

46. Pirapatdit S, Kishino E, Fujita K, Hashimoto H, Mori S, Saito S, et al. Dietary aLinked Galacto-Oligosaccharide Suppresses Ovalbumin-Induced Allergic Peritonitis in BALB/c Mice. Biosci Biotechnol Biochem. 2008;72(7):1901-7. 
47. Fujitani $\mathrm{S}$, Ueno K, Kamiya $\mathrm{T}$, Tsukahara $\mathrm{T}$, Ishihara $\mathrm{K}$, Kitabayashi $\mathrm{T}$, et al. Increased Number of CCR4-positive Cells in the Duodenum of Ovalbumininduced Food Allergy Model NC/jic Mice and Antiallergic Activity of Fructooligosaccharides. Allergol Int. 2007;56(2):131-8.

48. Vos AP, van Esch BC, Stahl B, M'Rabet L, Folkerts G, Nijkamp FP, et al. Dietary supplementation with specific oligosaccharide mixtures decreases parameters of allergic asthma in mice. Int Immunopharmacol. 2007;7(12):1582-7.

49. Hogenkamp A, Thijssen S, van Vlies N, Garssen J. Supplementing Pregnant Mice with a Specific Mixture of Nondigestible Oligosaccharides Reduces Symptoms of Allergic Asthma in Male Offspring. J Nutr. 2015;145(3):640-6.

50. Pascal Gourbeyre, Gall S Le, Champ M, Denery-papini S, Bodinier M. Exposure to a Galactooligosaccharides / Inulin Prebiotic Mix at Different Developmental Time Points Differentially Modulates Immune Responses in Mice. 2012;60:11942-51.

51. Gourbeyre P, Desbuards N, Grémy G, Tranquet O, Champ M, Denery-Papini $S$, et al. Perinatal and postweaning exposure to galactooligosaccharides/inulin prebiotics induced biomarkers linked to tolerance mechanism in a mouse model of strong allergic sensitization. J Agric Food Chem. 2013;61(26):631120.

52. Fujiwara R, Takemura N, Watanabe J, Sonoyama K. Maternal consumption of fructo-oligosaccharide diminishes the severity of skin inflammation in offspring of NC/Nga mice. Br J Nutr. 2010;103(4):530-8.

53. Bouchaud G, Castan L, Chesné J, Braza F, Aubert P, Neunlist M, et al. Maternal exposure to GOS/inulin mixture prevents food allergies and promotes tolerance in offspring in mice. Allergy Eur $\mathrm{J}$ Allergy Clin Immunol. 2016;71(1):68-76.

54. Osborn DA, Sinn JK. Prebiotics in infants for prevention of allergy. Cochrane Database Syst Rev. 2013;2013(3).

55. Cuello-Garcia C, Fiocchi A, Pawankar R, Yepes-Nuñez JJ, Morgano GP, Zhang $\mathrm{Y}$, et al. Prebiotics for the prevention of allergies: A systematic review and meta-analysis of randomized controlled trials. Clin Exp Allergy. 2017;47(11):1468-77.

56. Arslanoglu S, Moro GE, Schmitt J, Tandoi L, Rizzardi S, Boehm G. Early 
dietary intervention with a mixture of prebiotic oligosaccharides reduces the incidence of allergic manifestations and infections during the first two years of life. J Nutr. 2008;138(6):1091-5.

57. Grüber C, Van Stuijvenberg M, Mosca F, Moro G, Chirico G, Braegger CP, et al. Reduced occurrence of early atopic dermatitis because of immunoactive prebiotics among low-atopy-risk infants. J Allergy Clin Immunol. 2010;126(4):791-7.

58. Ivakhnenko OS, Nyankovskyy SL. Effect of the specific infant formula mixture of oligosaccharides on local immunity and development of allergic and infectious disease in young children: Randomized study. Pediatr Pol. 2013;88(5):398-404.

59. Ziegler E, Vanderhoof JA, Petschow B, Mitmesser SH, Stolz SI, Harris CL, et al. Term Infants Fed Formula Supplemented With Selected Blends of Prebiotics. J Pediatr Gastroenterol Nutr. 2007;44(3):359-64.

60. Boyle RJ, Tang MLK, Chiang WC, Chua MC, Ismail I, Nauta A, et al. Prebioticsupplemented partially hydrolysed cow's milk formula for the prevention of eczema in high-risk infants: A randomized controlled trial. Allergy Eur J Allergy Clin Immunol. 2016;71(5):701-10.

61. Yepes-Nuñez JJ, Fiocchi A, Pawankar R, Cuello-Garcia CA, Zhang Y, Morgano GP, et al. World Allergy Organization-McMaster University Guidelines for Allergic Disease Prevention (GLAD-P): Vitamin D. World Allergy Organ J. 2016;9(1):1-10. 


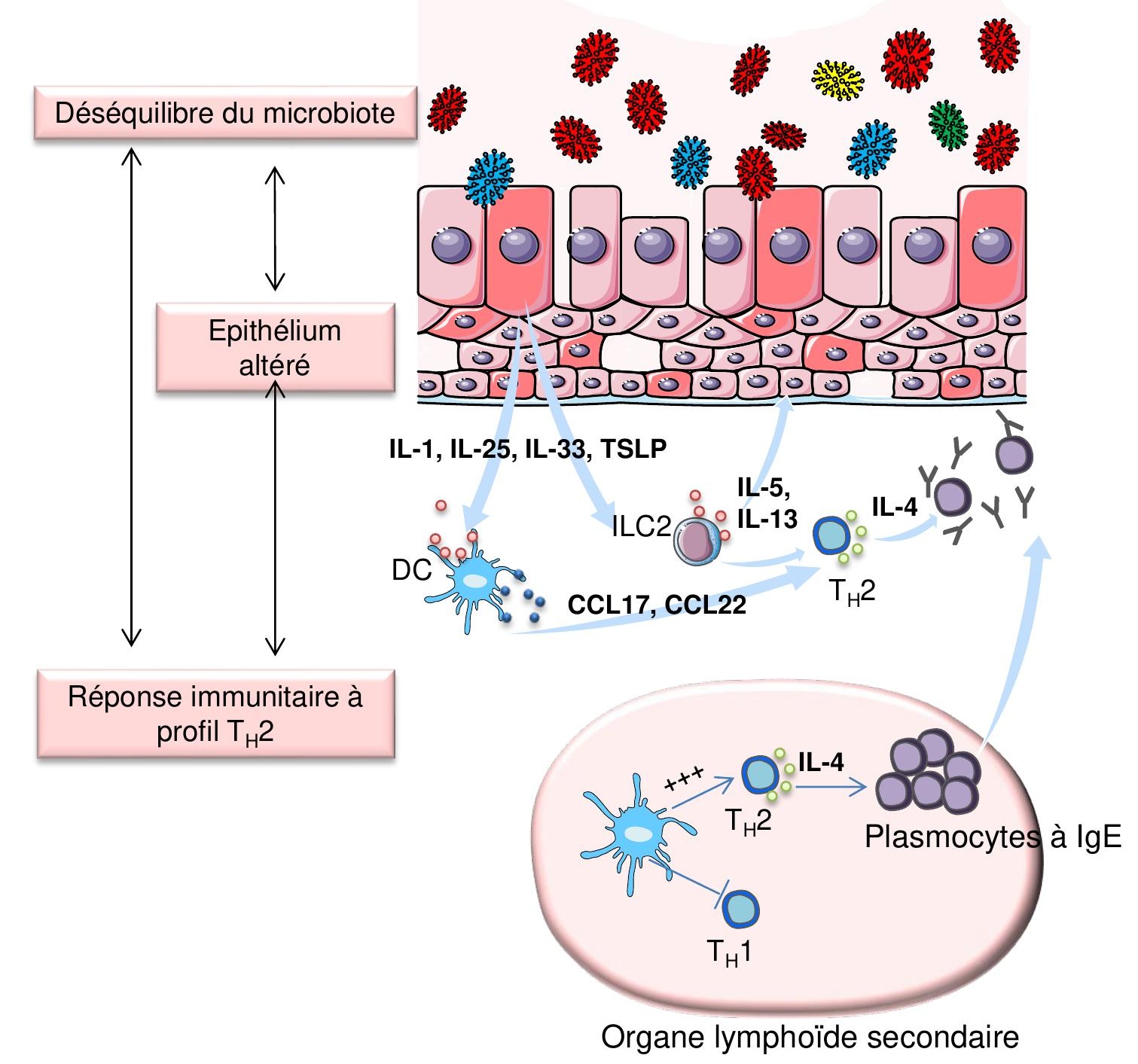

Figure 1: Représentation schématique des 3 acteurs biologiques impliqués dans le déclenchement de l'allergie.

Chez les patients allergiques, on assiste à une diminution de la diversité microbienne, à des modifications de la représentativité de certains groupes bactériens (en faveur des Firmicutes et en défaveur des Bifidobactéries par exemple) et à une altération de la fonctionnalité du microbiote (diminution de la quantité d'AGCC produits par les Bifidobactéries par exemple). On observe des altérations des épithéliums pulmonaire, cutanée ou intestinale. Les jonctions serrées, les villosités et d'autres paramètres physiologiques sont modifiés conduisant à une perméabilité aux allergènes augmentée. Les cellules épithéliales des patients allergiques présentent une sécrétion augmentée de cytokines inflammatoires (IL-1, IL25 , IL-33, TSLP). Les ILC2 et les DC présentent dans les épithelia vont répondre à ces signaux en sécrétant à l'heure tour des cytokines (IL-5, IL-13) et chémokines (CCL17, CCL22) qui vont orienter la réponse immunitaire vers un profil $\mathrm{T}_{\mathrm{H}} 2$. Notamment, cela permettra la prolifération de plasmocytes sécréteurs d'IgE, signature de l'allergie. La présence d'IgE permettra l'apparition des symptômes allergiques via la dégranulation des mastocytes et/ou basophiles. 


\section{Prébiotiques}

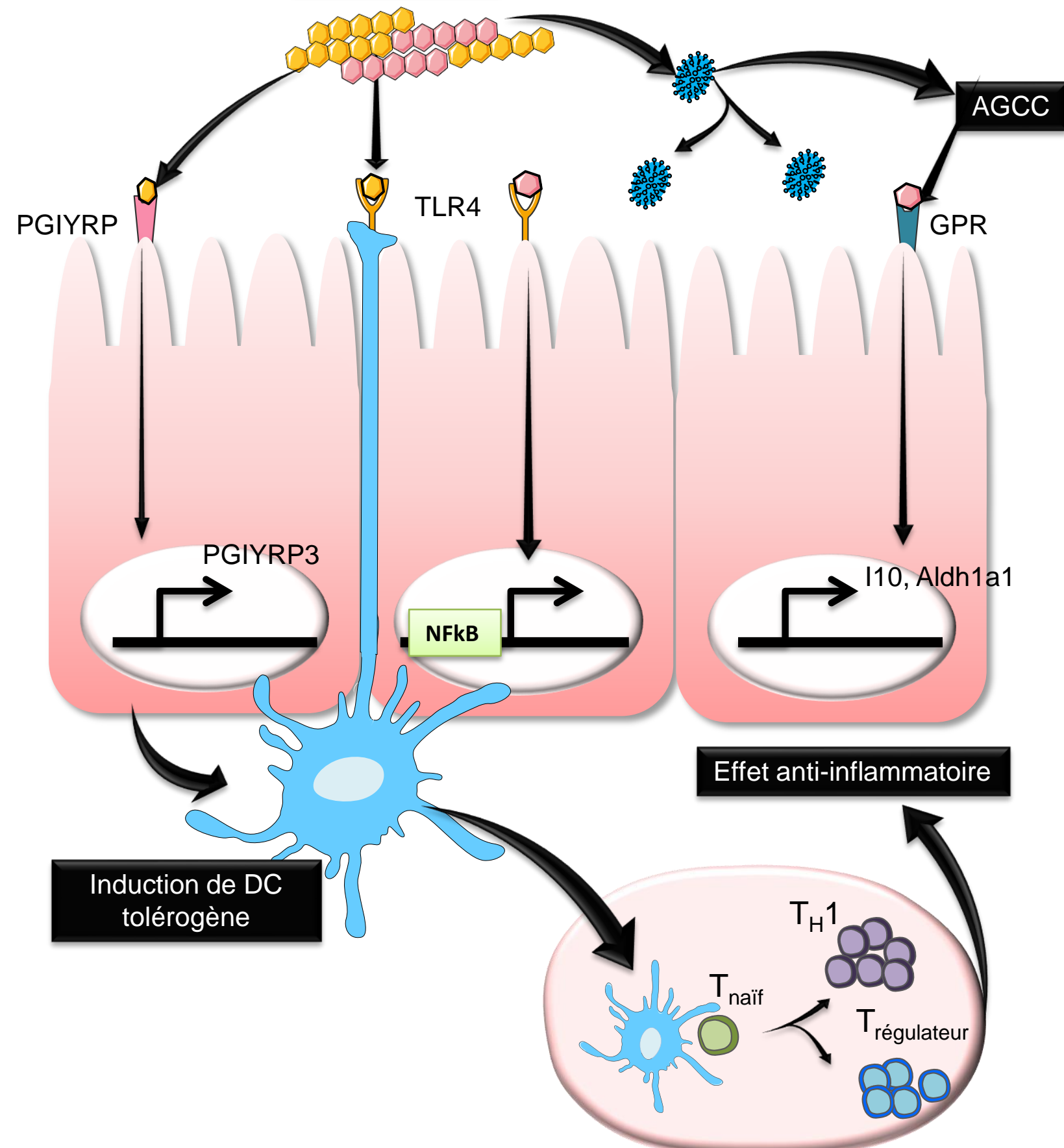

Figure 2. Effets direct et indirect des prébiotiques sur l'épithélium intestinal et le système immunitaire.

Certaines bactéries dites " bénéfiques 》 comme Bifidobactérium vont utiliser les prébiotiques comme substrat alimentaire. Leur consommation va induire la croissance de leur taxon ainsi que la production d'AGCC. Les $A G C C$ sont une véritable source d'énergie pour les cellules épithéliales intestinales. Les AGCC interviennent dans la régulation de l'expression de gènes en se liant à des récepteurs couplés à des protéines G : GPR41 (ou FFAR3) et GPR43 (ou FFAR2). Ainsi, la voie de signalisation induisant l'expression des protéines I10 et Aldh1a1 va être activée et les gènes impliqués dans la sécrétion du mucus et la prolifération cellulaire vont être également modulés. Les prébiotiques sont également capables d'avoir un impact direct sur les cellules épithéliale et cellules dendritiques en liant respectivement les récepteurs PGIYRP et TLR4. Ces liaisons permettront la transcription de gènes orientant les cellules dendritiques vers un profil tolérogène, qui une fois dans les ganglions mésentériques induiront une différenciation des cellules $T$ naïves vers des LT régulateurs, ayant un effet antiinflammatoire sur l'épithélium et donc protecteur. 


\begin{tabular}{|c|c|c|c|c|c|c|c|}
\hline & Année & Auteurs & Modèle & Prébiotiques utilisés & Age des animaux & Fenêtre de supplémentation & Résultats \\
\hline \multirow{6}{*}{ 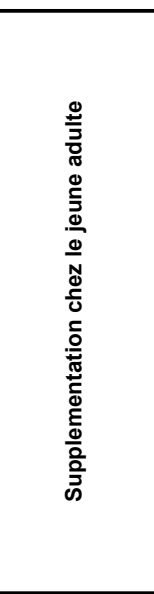 } & 2005 & $\begin{array}{l}\text { Kei Sonoyama, Hiroshi Watanabe, } \\
\text { Jun Watanabe, Natsu Yamaguchi, } \\
\text { \& al. (76) }\end{array}$ & Allergie respiratoire & $\begin{array}{l}50 \mathrm{~g} / \mathrm{kg} \text { Raffinose; } \\
\text { GOS; FOS; XOS }\end{array}$ & rat 5 semaines & 3 semaines & $\begin{array}{l}\text { Diminue l'infiltration des éosinophiles et des cellules Th2 lors de } \\
\text { l'induction d'une allergie respiratoire }\end{array}$ \\
\hline & 2007 & $\begin{array}{l}\text { A.P. Vos, B.C. van Esch, B. Stahl, } \\
\text { L. M'Rabet, G. Folkerts, F.P. } \\
\text { Nijkamp \& J. Garssen (61) }\end{array}$ & \begin{tabular}{|l} 
Asthme allergique \\
\end{tabular} & $\begin{array}{l}\text { 50g/kg scGOS:IcFOS } \\
(9: 1)+\text { pAOS }\end{array}$ & souris 5 à 8 semaines & 8 semaines & $\begin{array}{l}\text { La supplémentation alimentaire provoque une orientation de la } \\
\text { réponse immunitaire vers la réponse Th1 }\end{array}$ \\
\hline & 2007 & $\begin{array}{l}\text { Shinobu Fujitani, Kozo Ueno, } \\
\text { Taro Kamiya, Takamitsu } \\
\text { Tsukahara, \& al. (60) }\end{array}$ & Allergie alimentaire & $50 \mathrm{~g} / \mathrm{kg}$ FOS & souris 6 semaines & 8 semaines & $\begin{array}{l}\text { Diminution des cellules CCR4 positives et des mastocytes sur le } \\
\text { site d'induction de l'allergie lors du déclenchement de l'allergie } \\
\text { chez les souris nourries avec la supplémentation en FOS. }\end{array}$ \\
\hline & 2008 & $\begin{array}{l}\text { Jun Watanabe, Naho Sasajima, } \\
\text { Akiko Aramaki \& Kei Sonoyama } \\
\text { (58) }\end{array}$ & $\begin{array}{l}\text { Hypoersensibilité de } \\
\text { contact }\end{array}$ & $50 \mathrm{~g} / \mathrm{kg} \mathrm{FOS}$ & souris 5 semaines & 3 semaines & $\begin{array}{l}\text { L'hypersensibilité de contact est réduit par la supplémentation en } \\
\text { FOS. Le nombre de bifidobactéries intestinales était augmenté } \\
\text { par FOS, et B. pseudolongum était la plus représentées au sein } \\
\text { des bifidobactéries. }\end{array}$ \\
\hline & 2008 & $\begin{array}{l}\text { Pirapatdit S, Kishino E, Fujita K, } \\
\text { Hashimoto H, Mori S, Saito S, \& } \\
\text { al. (59) }\end{array}$ & Peritonite allergique & $50 \mathrm{~g} / \mathrm{kg}$ a-GOS & souris 4 semaines & 2 semaines & $\begin{array}{l}\text { Diminution de l'infiltration cellulaire lors d'un réaction allergique } \\
\text { par la diminution de la sécrétion de chimiokines } \\
\text { chemoattractantes dans la cavité péritonéale. }\end{array}$ \\
\hline & 2015 & $\begin{array}{l}\text { Kim AT Verheijden, Linette EM } \\
\text { Willemsen, Saskia Braber, Thea } \\
\text { Leusink-Muis et al. (75) }\end{array}$ & Allergie respiratoire & régime avec $1 \%$ de GOS & souris 6-8 semaines & 4 semaines & $\begin{array}{l}\text { Prévention de l'induction de l'éosinophilie des voies respiratoires } \\
\text { et de la sécrétion des cytokines et de chimiokines liées au profil } \\
\text { Th2 dans les poumons. }\end{array}$ \\
\hline \multirow{3}{*}{ 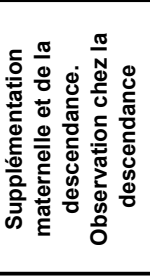 } & 2010 & $\begin{array}{l}\text { Reiko Fujiwara, Naoki Takemura, } \\
\text { Jun Watanabe and Kei Sonoyama } \\
\text { (65) }\end{array}$ & inflammation cutanée & $50 \mathrm{~g} / \mathrm{kg}$ FOS & souris 8 semaines & $\begin{array}{l}\text { Gestation + allaitement, et jusqu'à la fin du } \\
\text { protocole }\end{array}$ & $\begin{array}{l}\text { La consommation de prébiotiques diminue la gravité de la } \\
\text { dermatite atopique. }\end{array}$ \\
\hline & 2012 & \begin{tabular}{|l|} 
Pascal Gourbeyre, Nicolas \\
Desbuards, Guilaine Grémy, \\
Sophie Le Gall \& al. (63) \\
\end{tabular} & Allergie Alimentaire & 40g/kg GOS:Inuline (9:1) & souris 6 à 12 semaines & $\begin{array}{l}\begin{array}{l}\text { Gestation + allaitement, et jusqu'à la fin du } \\
\text { protocole }\end{array} \\
\end{array}$ & $\begin{array}{l}\text { La supplémentatation alimentaire stimule la tolérance immunitaire } \\
\text { et renforce la barrière intestinale. }\end{array}$ \\
\hline & 2013 & \begin{tabular}{|l|} 
Pascal Gourbeyre, Nicolas \\
Desbuards, Guilaine Grémy, \\
Olivier Tranquet, \& al. (64)
\end{tabular} & Allergie Alimentaire & 40g/kg GOS:Inuline (9:1) & souris 6 à 12 semaines & $\begin{array}{l}\text { Gestation + allaitement, et jusqu'à la fin du } \\
\text { protocole }\end{array}$ & $\begin{array}{l}\text { Augmentation des biomarqueurs de la tolérance après } \\
\text { sensibilisation. }\end{array}$ \\
\hline \multirow{4}{*}{ 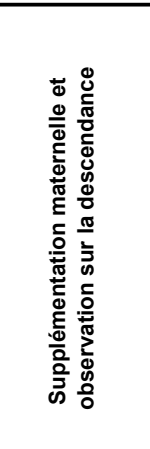 } & 2010 & $\begin{array}{l}\text { Reiko Fujiwara, Naoki Takemura, } \\
\text { Jun Watanabe and Kei Sonoyama } \\
\text { (65) }\end{array}$ & inflammation cutanée & $50 \mathrm{~g} / \mathrm{kg}$ FOS & souris 8 semaines & Gestation + allaitement & $\begin{array}{l}\text { La consommation de prébiotiques diminue la gravité de la } \\
\text { dermatite atopique. }\end{array}$ \\
\hline & 2015 & $\begin{array}{l}\text { Astrid Hogenkamp, Suzan } \\
\text { Thijssen, Naomi van Vlies, \& } \\
\text { Johan Garssen (62) }\end{array}$ & Asthme allergique & $\begin{array}{l}\text { 50g/kg scGOS:IcFOS } \\
(9: 1)\end{array}$ & souris 8 semaines & Gestation & $\begin{array}{l}\text { Diminuation de l'hyper-réactivité des voies aériennes, des IgE } \\
\text { spécifiques, augmentation des IgG2a spécifiques et des T } \\
\text { régulateurs au niveau périphérique. }\end{array}$ \\
\hline & 2015 & $\begin{array}{l}\text { Astrid Hogenkamp, Leon MJ } \\
\text { Knippels, Johan Garssen, and } \\
\text { Betty CAM van Esch (77) }\end{array}$ & Allergie Alimentaire & $\begin{array}{l}\text { 50g/kg scGOS:IcFOS } \\
(9: 1)\end{array}$ & souris 10 semaines & Gestation ou allaitement & Diminution des symptômes allergiques. \\
\hline & 2015 & \begin{tabular}{|l|} 
Bouchaud G, Castan L, Chesne J, \\
Braza F, Aubert P, Neunlist M, \\
Magnan A, Bodinier M. (66)
\end{tabular} & Allergie Alimentaire & 40g/kg GOS:Inuline (9:1) & souris 8 semaines & Gestation + allaitement & $\begin{array}{l}\text { Modification du microbiote, protection de la barrière intestinale et } \\
\text { induction d'une tolérance immunitaire }\end{array}$ \\
\hline
\end{tabular}


Tableau : Recencemment des études cliniques utilisant les prébiotiques chez I en ant pour la prévention des allergies.

\begin{tabular}{|c|c|c|c|c|c|c|c|}
\hline & Année & Auteurs & S mptomes & $\begin{array}{c}\text { Durée de la } \\
\text { supplémentations }\end{array}$ & cohorte & Prébiotiques & Résultats \\
\hline \multirow{6}{*}{ 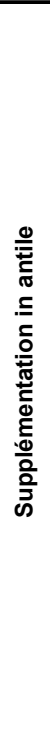 } & 2006 & $\begin{array}{l}\text { G Moro, S Arslanoglu, B Stahl, J } \\
\text { Jelinek, U Wahn, G Boehm (78) }\end{array}$ & $\begin{array}{l}\text { dermatite atopique } \\
\text { chez les enfants à haut } \\
\text { rique }\end{array}$ & 6 premier mois de vie & $\begin{array}{l}102 \text { infants in the } \\
\text { prebiotic group and } \\
104 \text { infants in the } \\
\text { placebo }\end{array}$ & GOS/FOS (9:1) $8 \mathrm{~g} / 1 \mathrm{~L}$ de lait & $\begin{array}{l}\text { le développement de la dermatite atopique au cours des si } \\
\text { premiers mois de vie a été significativement réduit par la } \\
\text { supplémentation. }\end{array}$ \\
\hline & 2008 & $\begin{array}{l}\text { Sertac Arslanoglu, Guido E. Moro, } \\
\text { Joachim Schmitt, Laura Tandoi, } \\
\text { Silvia Rizzardi, and Gunther } \\
\text { Boehm (69) }\end{array}$ & $\begin{array}{l}\text { dermatite atopique } \\
\text { chez les enfants à haut } \\
\text { rique }\end{array}$ & 6 premier mois de vie & $\begin{array}{l}68 \text { in placebo, } 66 \text { in } \\
\text { intervention group }\end{array}$ & GOS/FOS (9:1) 8 g/L de lait & $\begin{array}{l}\text { Au cours des } 2 \text { ans suivant la supplémentation, les manifestations } \\
\text { allergiques et les infections sont réduites dans le groupe } \\
\text { supplémenté. }\end{array}$ \\
\hline & 2012 & $\begin{array}{l}\text { S. ARSLANOGLU, , G.E. MORO, } \\
\text { G. BOEHM , F. WIENZ, B. STAHL } \\
\text { E. BERTINO (79) }\end{array}$ & $\begin{array}{l}\text { dermatite atopique } \\
\text { chez les enfants à haut } \\
\text { rique }\end{array}$ & 6 premier mois de vie & $\begin{array}{l}50 \text { in placebo group, } \\
42 \text { in intervention } \\
\text { group }\end{array}$ & GOS/FOS (9:1) $8 \mathrm{~g} / \mathrm{L}$ de lait & $\begin{array}{l}\text { Les manifestation de dermatite atopique ou de signes allergiques } \\
\text { au cours des } 5 \text { ans de vie ont été réduite significativement dans le } \\
\text { groupe supplémenté. }\end{array}$ \\
\hline & 2010 & $\begin{array}{l}\text { Christoph Gruber, Margriet van } \\
\text { Stuijvenberg, Fabio Mosca, Guido } \\
\text { Moro (70) }\end{array}$ & $\begin{array}{l}\text { dermatite atopique } \\
\text { chez les enfants à } \\
\text { faible risque }\end{array}$ & à partir de 2 mois de vie & $\begin{array}{l}414 \text { infants were } \\
\text { randomized to the } \\
\text { prebiotic group and } \\
416 \text { infants to the } \\
\text { control group }\end{array}$ & $\begin{array}{l}\text { scGOS/lcFOS }(9: 1 ; 6,8 \mathrm{~g} / \mathrm{L})+ \\
\text { pAOS }(1,2 \mathrm{~g} / \mathrm{L}) \text { de lait }\end{array}$ & $\begin{array}{l}\text { A l' ge de } 1 \text { an, les enfants alimentés avec la formule en lait } \\
\text { enrichie en prébiotiques ont moins développé de dermatite } \\
\text { atopique. }\end{array}$ \\
\hline & 2013 & $\begin{array}{l}\text { Olena S. Ivakhnenko, Serhiy L. } \\
\text { Nyankovskyy (71) }\end{array}$ & $\begin{array}{l}\text { Développement de } \\
\text { maladies infectieuses } \\
\text { et d'allergies }\end{array}$ & 1er mois de vie & $\begin{array}{l}80 \text { enfants allaités, } \\
80 \text { enfants sous lait } \\
\text { supplémenté et } 80 \\
\text { enfant sous lait } \\
\text { standard }\end{array}$ & scGOS/lcFOS; (9:1) $8 \mathrm{~g} / \mathrm{L}$ & $\begin{array}{l}\text { Diminution du développement des maladies infectieuses et des } \\
\text { allergies, notamment alimentaires, chez les enfants nourris avec le } \\
\text { lait supplémenté en prébiotiques. }\end{array}$ \\
\hline & 2016 & $\begin{array}{l}\text { R. J. Boyle, M. L.-K. Tang, W. C. } \\
\text { Chiang, M. C. Chua, I. Ismail, A. } \\
\text { Nauta, J. O B. Hourihane (73) }\end{array}$ & \begin{tabular}{|l|} 
Eczéma chez les \\
enfant à haut risque
\end{tabular} & $\begin{array}{l}\text { jusqu'à l' ge de } 6 \text { mois si } \\
\text { la préparation a été } \\
\text { introduite avant } 18 \\
\text { semaines }\end{array}$ & 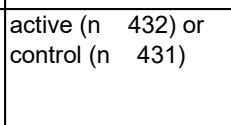 & $8 \mathrm{~g} / \mathrm{L} \mathrm{pHF-OS}$ & $\begin{array}{l}\text { pHF-OS ne prévient pas l'eczéma la première année chez les } \\
\text { nourrissons à haut risque. }\end{array}$ \\
\hline
\end{tabular}

